\title{
Silicone Breast Implant Applied to Blepharoplasty Can Help Effective Compression Hemostasis: A Simple and Effective Method
}

\author{
Tingfeng Xiong ${ }^{1}$ (D) $\cdot$ Yangze Ou$^{1}$
}

Received: 20 November 2019/Accepted: 21 November 2019/Published online: 2 December 2019

(C) Springer Science+Business Media, LLC, part of Springer Nature and International Society of Aesthetic Plastic Surgery 2019

Level of Evidence $V$ This journal requires that authors assign a level of evidence to each article. For a full description of these Evidence-Based Medicine ratings, please refer to the Table of Contents or the online Instructions to Authors www.springer.com/00266.

Dear Editor,

Intraoperative bleeding during blepharoplasty often affects the operator's field of vision. It also results in longer recovery times for the patient. Therefore, less intraoperative bleeding is critical.

For blepharoplasty, when bleeding on one side, we can perform contralateral surgery first and at the same time perform compression hemostasis. When the contralateral operation is completed, the side of the bleeding often stops bleeding. Thus, we avoided the time period of bleeding and reduced the operation time. Usually, when we are operating, the assistant uses the hand to press one side of the eye, and the operator performs the other side. But we sometimes lack assistants, or the assistant is inexperienced, overstressing causes patient discomfort and insufficient compression can lead to bleeding. So, in order to solve this problem, we need to find a material that provides moderate pressure to help oppress the eye. Initially we thought of using water: Tighten the water into the glove and fasten it and then insert the two layers of sterile gloves. However, due to the fluidity of the water, it is easy to slide, and the water easily contaminates the wound after the glove is broken. Therefore, we abandoned this method and sought more stable equipment. Finally, we used a silicone breast implant instead of water. The content of the silicone is difficult to break, and it does not roll easily. Then, we used a silicone breast implant to assist blepharoplasty surgery: When performing one side of the procedure, the prosthesis is placed on the other side of the eye, and the operator uses the ipsilateral hand to slightly compress the prosthesis to make the pressure moderate (so the size of the prosthesis is not strictly required). Therefore, the surgeon can perform one side surgery well while pressing the other side to reduce bleeding.

This is the first time we have found that using this method to assist blepharoplasty, the surgical results are satisfactory, and the materials are convenient, safe and effective, and worthy of promotion. Experienced surgeons can even perform surgery alone when performing blepharoplasty and encounter little bleeding.

\section{Compliance with Ethical Standards}

Conflict of interest The author has no financial interest to declare in relation to the content of this communication.

Human and Animal Rights This article does not contain any studies with human participants or animals performed by any of the authors.

Informed Consent For this type of study, informed consent is not required.
Tingfeng Xiong

Tinfine@outlook.com

1 Department of Plastic Surgery, Affiliated Hospital of JiuJiang University, No. 57 Xun yang East Road, Jiujiang 332000, Jiangxi, China 